\title{
Malignant Transformation of an HNF1a-Inactivated Hepatocellular Adenoma to Hepatocellular Carcinoma
}

\author{
Joris T. Hepkema ${ }^{a}$ Floris B. Poelmann ${ }^{a}$ Annette S.H. Gouw \\ Robbert J. de Haas ${ }^{c}$ Evelien W. Duiker ${ }^{b}$ Hans Blokzijl ${ }^{d}$ \\ Joost M. Klaase ${ }^{a}$
}

${ }^{a}$ Division of Hepato-Pancreato-Biliary Surgery and Liver Transplantation, Department of Surgery, University Medical Center Groningen, University of Groningen, Groningen, The Netherlands; ${ }^{b}$ Department of Medical Biology and Pathology, University Medical Center Groningen, University of Groningen, Groningen, The Netherlands; 'Department of Radiology, University Medical Center Groningen, University of Groningen, Groningen, The Netherlands; dDepartment of Gastroenterology and Hepatology, University Medical Center Groningen, University of Groningen, Groningen, The Netherlands

\section{Keywords}

Hepatocellular adenoma - Hepatocellular carcinoma - Hepatocyte nuclear factor 1-alpha . Imaging · Surgical resection · Laparoscopy
Abstract
Hepatocellular adenomas (HCA) are rare benign tumors of the liver, occurring predominantly in females using oral contraceptives. Our case describes a 66-year-old woman presenting with a palpable mass in her upper abdomen. Contrast-enhanced computed tomography and mag- netic resonance imaging showed a large exophytic mass protruding from the caudal border of liver segments IV and V, without visible metastases. Laparoscopic resection of the tumor and 


\section{Case Reports in Gastroenterology}

Case Rep Gastroenterol 2020;14:577-585

DOI: $10.1159 / 000510215$

(c) 2020 The Author(s). Published by S. Karger AG, Basel www.karger.com/crg

Hepkema et al.: Malignant Transformation of an HNF1a-Inactivated Hepatocellular Adenoma to Hepatocellular Carcinoma

gallbladder was performed. Histopathological examination showed a hepatocellular carcinoma with areas of HNF1a-HCA (H-HCA). This case shows that malignant transformation is possible in $\mathrm{H}-\mathrm{HCA}$. We present our preoperative decision-making process, as well as the role of imaging techniques in this rare case.

\section{Introduction}

Hepatocellular adenomas (HCA) are rare, benign tumors of the liver [1]. They occur predominantly in females using oral contraceptives [2, 3]. Four different subtypes of HCA have been described, with differing histopathological, immunopathological, and molecular characteristics. Overall, HCA have a relatively low chance of about $4.2 \%$ to become malignant [4]. However, HCA larger than $50 \mathrm{~mm}$ have a hemorrhagic risk of $20-25 \%$, as well as an increased risk of malignancy [5]. Usually, because of these risks, HCA of this size are removed surgically $[4,6]$. Among the four different subtypes of HCA, beta-catenin-activated HCA are known to carry the highest risk of malignant transformation, while hepatocyte nuclear factor 1-alpha (HNF1a)-inactivated HCA (H-HCA) almost never become malignant [1].

To aid in the understanding of the pathological mechanism behind these rare cases of $\mathrm{H}$ HCA transformation into hepatocellular carcinoma (HCC), we describe a patient with an HCC derived from an H-HCA. The current case has already been described as part of the study by Putra et al. [7]. However, their primary focus was on the histopathological and immunopathological aspects of the case series. The current case report will address the preoperative decision-making process and the role of imaging techniques in this rare case.

\section{Case Presentation}

A 66-year-old woman was referred to our tertiary referral center because of a liver tumor suspicious for malignancy. The patient did not have any known pre-existing liver disease. She did not use oral contraceptives. She presented at the outpatient clinic with a palpable mass in the right upper quadrant of the abdomen, without any other complaints. Notably, she did not experience any pain and was in good condition (physical status was classified as ASA2). Physical examination revealed a morbidly obese woman, BMI $35.6 \mathrm{~kg} / \mathrm{m}^{2}$, without signs of illness besides the palpable mass in the right upper quadrant of the abdomen. Liver function tests (alanine transaminase, aspartate transaminase, alkaline phosphatase, albumin, total protein, bilirubin, gamma-glutamyltransferase, and prothrombin time) and tumor marker results (alpha fetoprotein, carcinoembryonic antigen, and carbohydrate antigen 19-9) were normal. Hepatitis serology was initiated and showed a history of hepatitis C and E, but there was no active disease confirmed by a negative viral load determined by polymerase chain reaction. Contrast-enhanced computed tomography (CT) and magnetic resonance imaging (MRI) showed a solitary large (about $15 \times 20 \mathrm{~cm}$ ), heterogeneous exophytic mass protruding from the caudal border of liver segments IV and V with a heterogeneous hypervascular enhancement pattern, without clear washout in the portal venous or delayed contrast phases (Fig. 1). At MRI, the lesion showed a heterogeneous increased signal intensity at T2-weighted images, 


\section{Case Reports in Gastroenterology}

Case Rep Gastroenterol 2020;14:577-585

DOI: $10.1159 / 000510215$

(c) 2020 The Author(s). Published by S. Karger AG, Basel www.karger.com/crg

Hepkema et al.: Malignant Transformation of an HNF1a-Inactivated Hepatocellular Adenoma to Hepatocellular Carcinoma

and no intracellular fat was observed. In addition, no clear signs of steatosis, cirrhosis, or portal hypertension were present, and no metastases were found. Therefore, at imaging the diagnosis remained unclear (Table 1), and most importantly, malignancy (such as HCC or angiosarcoma) could not be ruled out.

A fibroscan was performed and showed a result of $6.0 \mathrm{Kpa}$, corresponding to F0 fibrosis, indicating no liver fibrosis. It also showed a controlled attenuation parameter of $310 \mathrm{db} / \mathrm{m}$, corresponding to severe steatosis (hepatocytes containing fat of $>66 \%$ ) [8]. After combining all of the aforementioned results, the differential diagnosis included HCC and angiosarcoma. After reviewing the case in a multidisciplinary liver tumor meeting, the lesion was considered most likely to be an HCC in a non-cirrhotic liver. This was in particular based on its heterogeneous T2-hyperintensity at MRI, combined with its hypervascular aspect. Then, it was decided to perform a combined laparoscopic resection of the tumor and gallbladder because of the close relationship between tumor and gallbladder, and because of the increased technical risk of a cholecystectomy at a later date, should the need arise. The decision to perform surgery was based on the risk of malignancy, as well as size. Although the tumor was large, a radical resection through a laparoscopic approach seemed both possible and feasible in experienced hands.

\section{Surgical Method}

We performed a laparoscopy, with the patient in the French position. The tumor was identified, measuring about $15 \times 20 \mathrm{~cm}$, originating from the caudal border of liver segments IV and V. No signs of peritoneal deposits were seen, and the liver showed no further abnormalities. A plane was established between gallbladder and tumor. Using the sealing technique and a tri-stapler, the tumor was dissected at the edge of the liver. The gallbladder was removed according to the Critical View of Safety (CVS) principles. There were no intraoperative signs of biliary leakage. The tumor and gallbladder were removed with an extraction bag through a small lower midline laparotomy. Both the tumor and the gallbladder were sent to the pathology department for examination. The exophytic tumor weighed 1,200 g (Fig. 2a). The postoperative course was uneventful, and the patient was discharged after 4 days. Two weeks after the operation, the patient was seen at the outpatient clinic and showed complete recovery without any complications.

\section{Pathology}

Pathological findings are shown in Figure 2b. Gross examination showed a multinodular lesion with necrotic and hemorrhagic areas. Some non-lesional liver tissue was present at the resection margin. Microscopic examination confirmed hemorrhage and necrosis. The lesion showed the characteristics of an HCC consisting of hepatocytes arranged in trabecular and pseudo-glandular structures, lined by CD34-positive endothelium and apparent reticulin loss. The tumor hepatocytes showed extensive steatosis, some anisonucleosis, and prominent nucleoli. There was no angioinvasion, and the resection margin was free from tumor.

Between the overt HCC areas, transitions to small areas compatible with HCA were observed. The HCA areas showed solitary arteries with absence of portal tracts, while reticulin loss, cellular atypia, or trabecular or pseudoglandular architecture were not present, together with a decrease in steatosis. 


\section{Case Reports in Gastroenterology}

Case Rep Gastroenterol 2020;14:577-585

DOI: $10.1159 / 000510215$
2020 The Author(s). Published by S. Karger AG, Base www.karger.com/crg

Hepkema et al.: Malignant Transformation of an HNF1a-Inactivated Hepatocellular Adenoma to Hepatocellular Carcinoma

Immunohistochemistry showed absence of LFABP in both HCC and HCA whilst the nonlesional liver tissue showed a normal diffuse expression pattern (Fig. 3). Amyloid AA and CRP were negative, and there was no nuclear b-catenin. Glutamine synthetase was diffusely expressed, stronger in the HCC than in the HCA. Mutational analysis showed GNAS exon 8 mutation in both HCA and HCC components.

\section{Discussion}

In this report, we have shown malignant transformation of H-HCA into HCC. In contrast to beta catenin-activated HCA, H-HCA has been very infrequently associated with malignancy and is the predominant type of HCA in liver adenomatosis. In patients with hepatic adenomatosis, H-HCA is most frequently seen [9]. The European Association for the Study of the Liver (EASL) guidelines, concerning benign liver tumors, currently recommend basing the management of patients with multiple HCA upon the size of the largest tumor [6]. Individuals with unilobular disease can be treated with hepatic resection, and for those with more widespread HCA, resection of the largest adenomas may be an option. Another method to achieve mass reduction of adenomas is embolization. In the most severe cases, resection of all large masses is not possible, and a liver transplantation should be considered an option. Our case supports the resection of large tumors suspected to be either HCA or HCC, since even H-HCA is now proven to carry a risk of malignant transformation, a quality that was still under debate.

In conclusion, malignant transformation of H-HCA into HCC is a rare entity that should be considered in patients with liver tumors measuring $>50 \mathrm{~mm}$. When encountered, laparoscopic resection of an exophytic mass is feasible.

\section{Acknowledgement}

The authors would like to thank everyone working at the UMCG involved in the care for the patient described in this case report.

\section{Statement of Ethics}

Ethical approval was not required for this article, as it is a case report and no aspects of the care of the patient were altered for the purpose of this case report. Written informed consent was obtained from the patient for publication of this case report and any accompanying images.

\section{Conflict of Interest Statement}

The authors have no conflicts of interest to declare. 


\section{Case Reports in Gastroenterology}

Case Rep Gastroenterol 2020;14:577-585

\begin{tabular}{l|l}
\hline DOI: $10.1159 / 000510215$ & $\begin{array}{l}\text { (c) } 2020 \text { The Author(s). Published by S. Karger AG, Basel } \\
\text { www.karger.com/crg }\end{array}$
\end{tabular}

Hepkema et al.: Malignant Transformation of an HNF1a-Inactivated Hepatocellular Adenoma to Hepatocellular Carcinoma

\section{Funding Sources}

No funding was required for the writing of this case report.

\section{Author Contributions}

All authors made substantial contributions to the conception and design of the study, analysis and interpretation of data, drafting the article, and revising it critically for important intellectual content. All authors gave their final approval of the version to be submitted. The acquisition of data was done by Dr. Poelmann.

\section{References}

1 Bioulac-Sage P, Sempoux C, Balabaud C. Hepatocellular Adenomas: morphology and Genomics. Gastroenterol Clin North Am. 2017 Jun;46(2):253-72.

2 Haring MP, Gouw AS, de Haas RJ, Cuperus FJ, de Jong KP, de Meijer VE. The effect of oral contraceptive pill cessation on hepatocellular adenoma diameter: A retrospective cohort study. Liver Int. 2019 May;39(5):905-13.

3 Perret AG, Mosnier JF, Porcheron J, Cuilleron M, Berthoux P, Boucheron S, et al. Role of oral contraceptives in the growth of a multilobular adenoma associated with a hepatocellular carcinoma in a young woman. J Hepatol. 1996 Dec;25(6):976-9.

4 Stoot JH, Coelen RJ, De Jong MC, Dejong CH. Malignant transformation of hepatocellular adenomas into hepatocellular carcinomas: a systematic review including more than 1600 adenoma cases. HPB (Oxford). 2010 Oct;12(8):509-22.

5 Dokmak S, Paradis V, Vilgrain V, Sauvanet A, Farges O, Valla D, et al. A single-center surgical experience of 122 patients with single and multiple hepatocellular adenomas. Gastroenterology. 2009 Nov;137(5):1698705.

6 Colombo M, Forner A, IJzermans J, Paradis V, Reeves H, Vilgrain V, et al.; European Association for the Study of the Liver (EASL). EASL Clinical Practice Guidelines on the management of benign liver tumours. J Hepatol. 2016 Aug;65(2):386-98.

7 Putra J, Ferrell LD, Gouw AS, Paradis V, Rishi A, Sempoux C, et al. Malignant transformation of liver fatty acid binding protein-deficient hepatocellular adenomas: histopathologic spectrum of a rare phenomenon. Mod Pathol. 2019 Apr;33(4):665-75.

8 Mikolasevic I, Orlic L, Franjic N, Hauser G, Stimac D, Milic S. Transient elastography (FibroScan(®)) with controlled attenuation parameter in the assessment of liver steatosis and fibrosis in patients with nonalcoholic fatty liver disease - Where do we stand? World J Gastroenterol. 2016 Aug;22(32):7236-51.

9 Barbier L, Nault JC, Dujardin F, Scotto B, Besson M, de Muret A, et al. Natural history of liver adenomatosis: A long-term observational study. J Hepatol. 2019 Dec;71(6):1184-92. 


\section{Case Reports in Gastroenterology}

Case Rep Gastroenterol 2020;14:577-585
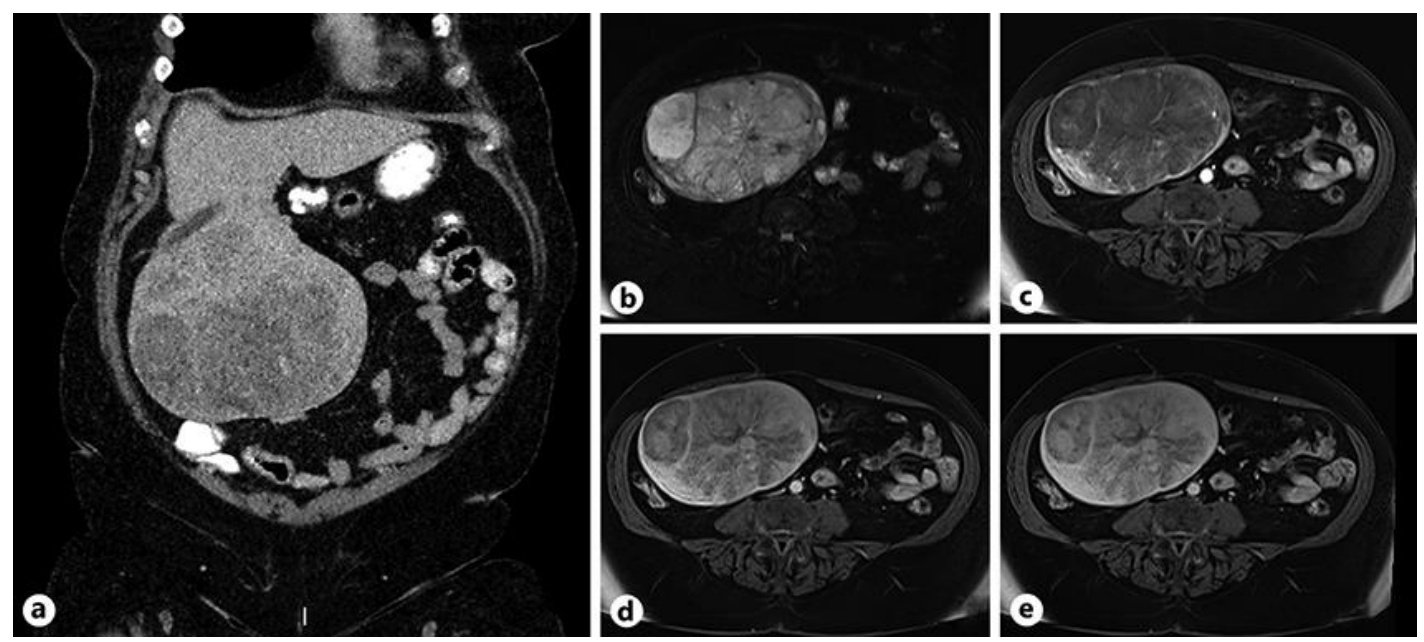

Fig. 1. CT and MRI of the exophytic liver tumor. a Coronal contrast-enhanced CT image of the abdomen at the portal venous phase showing the large exophytic mass originating from the caudal border of liver segments $4 \mathrm{~B}$ and 5 with a heterogeneous enhancement pattern. $\mathbf{b}$ Axial fat-suppressed T2-weighted MR image showing the large tumor with heterogeneous hyperintense signal intensity. c-e Axial fat-suppressed T1weighted MR images at the arterial (c), portal venous (d), and delayed phase (e), showing slight heterogeneous, predominantly peripheral arterial enhancement, with increasing enhancement at the portal venous and delayed phases. No significant drop of signal intensity was present at the opposed-phase T1-weighted images (not shown). 


\section{Case Reports in Gastroenterology}

Case Rep Gastroenterol 2020;14:577-585
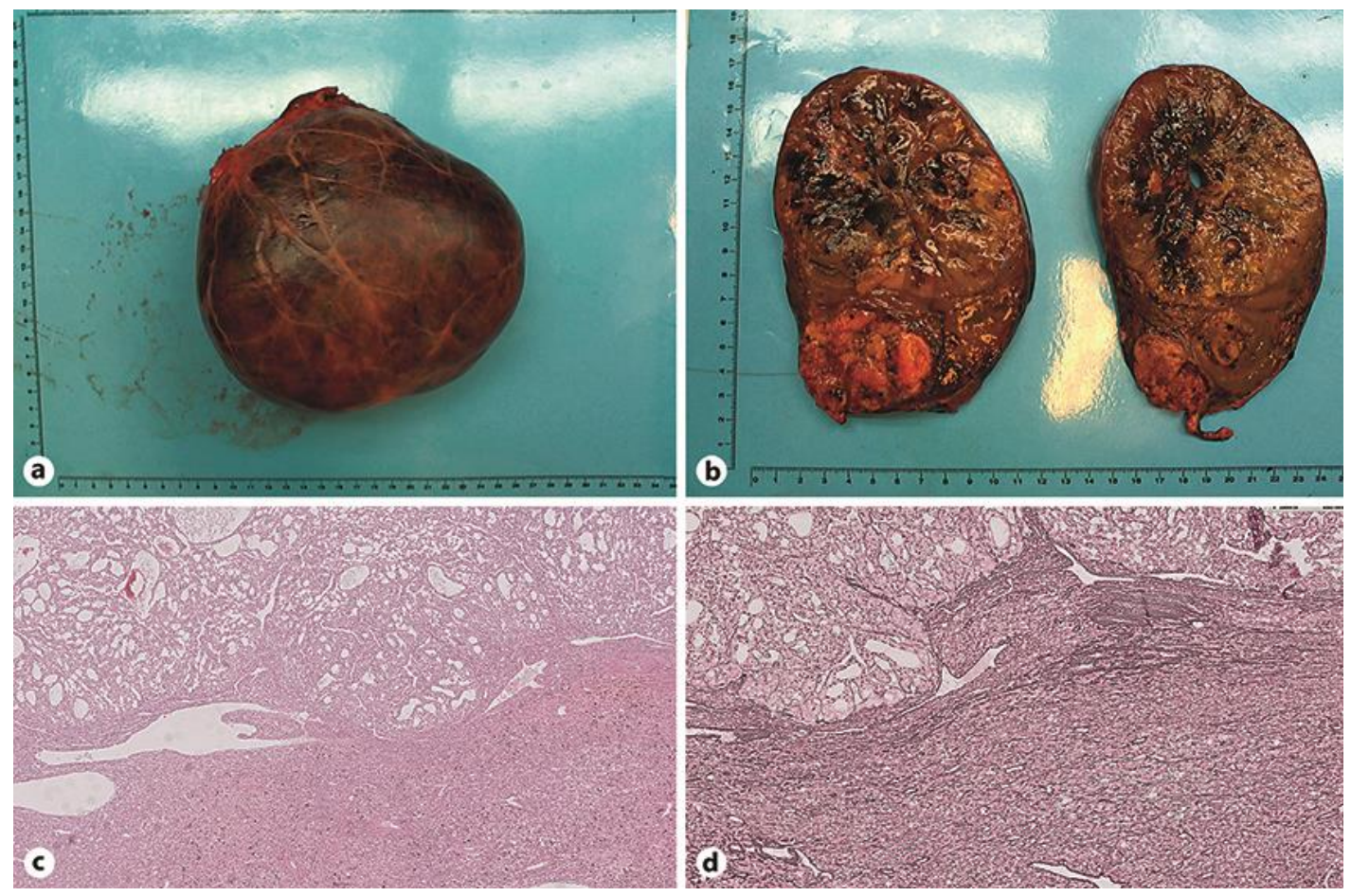

Fig. 2. Macroscopic aspect. Fresh resection specimen whole (a) and sliced (b). Almost the whole specimen was lesional and consisted of a multinodular tumor with partial myxoid/necrotic aspect and partial capsule formation. The different nodules were microscopically not clearly demarcated in HCC or adenoma, with an intimate mixture of the two components. Microscopic aspect. c Both components present, with the HCC component on top of the image and the adenomatous component at the bottom. The HCC is characterized by trabecular and pseudoglandular structures, while the adenomatous component consists of atrophic hepatocytes with some sinusoidal dilatation while unpaired arteries are present and portal tracts are absent (HE. $\times 10)$. $\mathbf{d}$ The same area in the reticulin stain. Note the disrupted reticulin framework and the thickened pseudoglandular structures in the HCC component, while the reticulin framework is preserved in the adenoma component. 


\section{Case Reports in Gastroenterology}
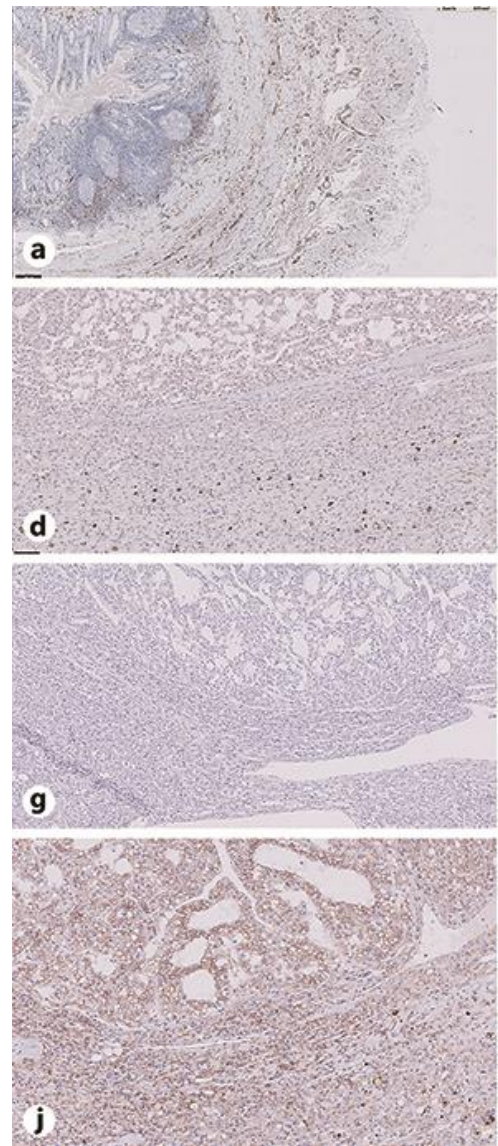

Case Rep Gastroenterol 2020;14:577-585

(C) 2020 The Author(s). Published by S. Karger AG, Basel www.karger.com/crg

Hepkema et al.: Malignant Transformation of an HNF1a-Inactivated Hepatocellular Adenoma to Hepatocellular Carcinoma
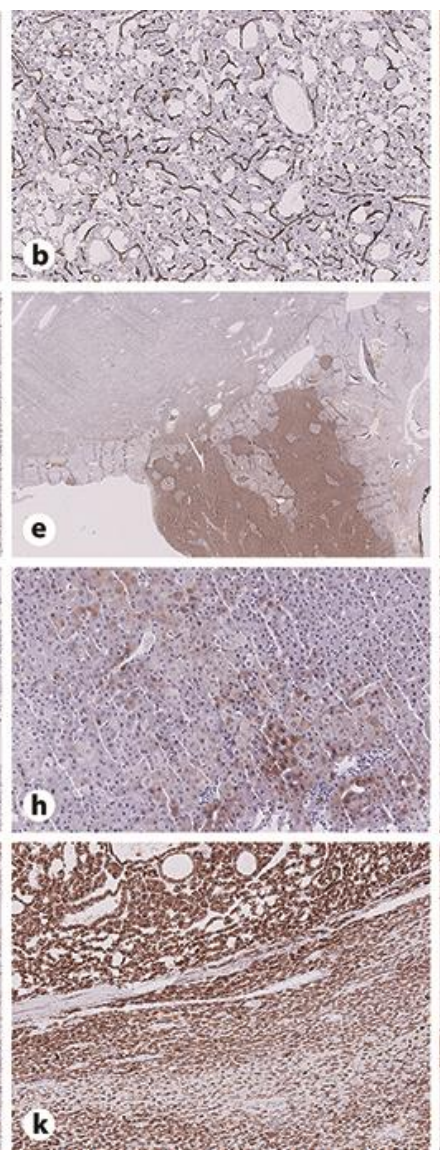
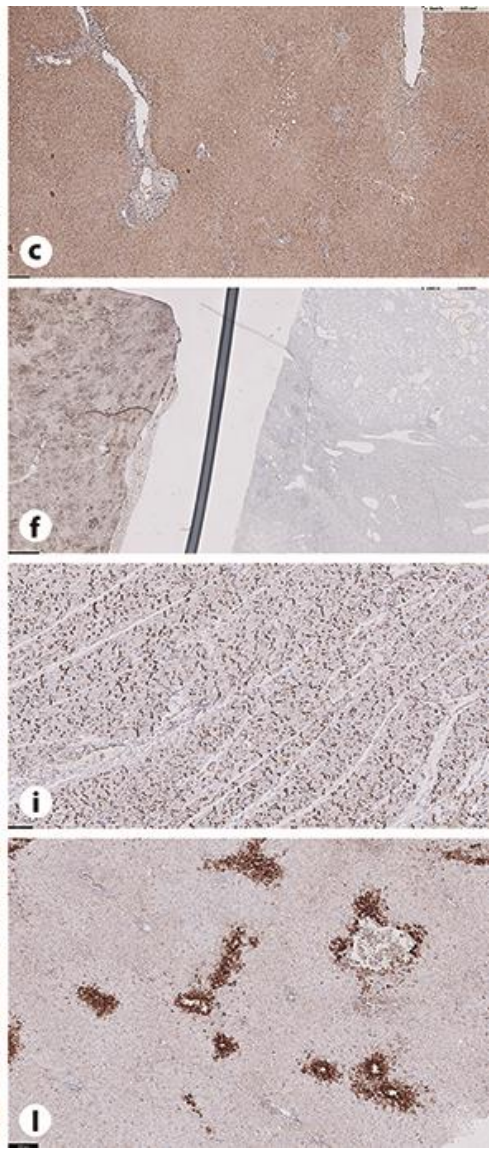

Fig. 3. Microscopic aspect with staining. Images were acquired through digital pathology. Objective is shown. a CD34 control staining at $\times 5$. $\mathbf{b}$ CD34 staining at $\times 5$. $\mathbf{c}$ LFABP control staining at $\times 5$. $\mathbf{d}$ LFABP staining of the HCC at $\times 10$. e LFABP staining of the border between lesional and non-lesional tissue at $\times 1$. $\mathbf{f}$ Amyloid-AA staining with control at $\times 1$. $\mathrm{g}$ CRP staining at $\times 5$. $\mathbf{h}$ CRP control staining at $\times 10$. $\mathbf{i}$ Beta-catenin control staining at $\times 10$. $\mathbf{j}$ Beta-catenin staining at $\times 10$. $\mathbf{k}$ Glutamine staining at $\times 5$. I Glutamine control staining at $\times 5$. 


\section{Case Reports in Gastroenterology}

DOI: 10.1159/000510215

(C) 2020 The Author(s). Published by S. Karger AG, Basel www.karger.com/crg

Hepkema et al.: Malignant Transformation of an HNF1a-Inactivated Hepatocellular Adenoma to Hepatocellular Carcinoma

Table 1. Interpretation of findings on CT and MRI

Findings

HCC

HCA

Large, heterogeneous lesion

Increased signal intensity at T2-weighted images

Absence of intracellular fat

Absence of cirrhosis

No washout in the portal venous or delayed contrast phases

$+\quad+/-$

$+$

$+/-$

$+/-$

$-$

+, increases suspicion; -, decreases suspicion. 